\title{
Self Field Measurements by Hall Sensors on the SeCRETS Short Sample CICC's Subjected to Cyclic Load
}

\author{
Yu. A. Ilyin, A. Nijhuis, H. H. J. ten Kate, B. Stepanov, and P. Bruzzone
}

\begin{abstract}
An imbalance in the transport current among the strands of a Cable-in-Conduit conductors (CICC) can be associated with the change of their performance. In order to understand and improve the performance of CICC's, it is essential to study the current imbalance. This paper focuses on the study of the current imbalance in two short samples of the SeCRETS (Segregated Copper Ratio Experiment on Transient Stability) conductors subjected to a cyclic load in the SULTAN facility. The self field around the conductors was measured on four locations by 32 miniature Hall sensors for a reconstruction of the current distribution. The results of the self field measurements in the DC tests are presented and discussed.
\end{abstract}

Index Terms-Cable-in-conduit conductor, current imbalance, self field measurements.

\section{INTRODUCTION}

$\mathbf{T}$ HE MAIN goal of the experiment with the SeCRETS short samples is to study the effect of an electromagnetic cyclic load (at constant background field, ramping the current up to its critical value and back to zero with a constant rate) on the performance of both conductors, identical in the overall cross-section except for the distribution of the copper stabilizer [1]. The voltage-current $(V-I)$ and voltage-temperature $(V-T)$ characteristics, AC losses, stability and current imbalance were measured before and after cyclic loads. As the result, the change of the conductors' performance characteristics (critical current, current sharing temperature and $n$-factor) with the number of the load cycles was observed [2].

From the perspective of this result a study of the current distribution would be helpful to clarify whether or not the observed change could be correlated to a difference in the current distribution inside the conductors. For the purpose of the current reconstruction, the self field around the conductors was measured on four locations by miniature Hall sensors (HS) combined in annular arrays.

The results of the self field measurements during the $V-T$ tests are presented and compared with the earlier obtained results of the application of the HS method to study the current imbalance in the SeCRETS long sample [3].

Manuscript received August 5, 2002. This work was supported in part by the EU under Contract EFDA-00/552.

Yu. A. Ilyin, A. Nijhuis, and H. H. J. ten Kate are with the University of Twente, Department of Applied Physics, 7500AE Enschede, the Netherlands (e-mail: y.ilyin@tn.utwente.nl).

B. Stepanov and P. Bruzzone are with CRPP - Technologie de la Fusion, CH-5232 Villigen-PSI, Switzerland (e-mail: pierluigi.bruzzone@ psi.ch).

Digital Object Identifier 10.1109/TASC.2003.812880

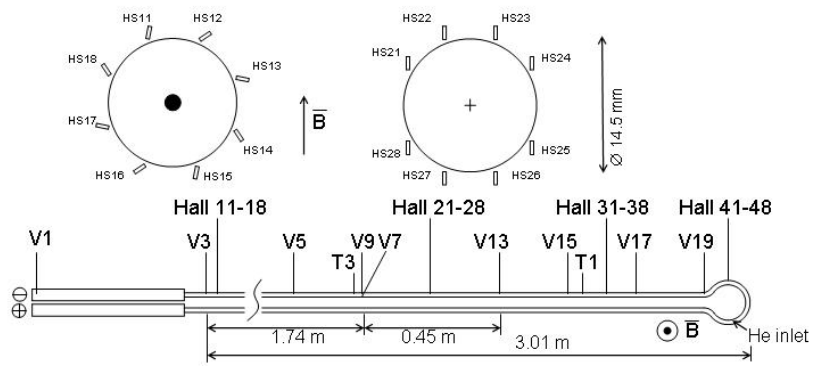

Fig. 1. The layout of the U-shaped sample with locations of the voltage taps and sensors on one leg of the sample. Also shown is the orientation of the HS's in the arrays near the joints and in the high field region in respect to the directions of the transport current and SULTAN field.

\section{SAMPLES AND INSTRUMENTATION}

Both short SeCRETS samples $\left(A-144 \mathrm{Nb}_{3} \mathrm{Sn}\right.$ strands, is typical ITER, high grade, last but one cable stage; $B-112 \mathrm{Nb}_{3} \mathrm{Sn}$ plus $16 \mathrm{Cu}$ strands) are prepared identically, hairpin-shaped with a length of the straight parts of about $3 \mathrm{~m}$. Each sample is instrumented with in total 24 potential taps to measure the longitudinal and transversal voltages. In addition 5 temperature sensors, 4 heaters and 4 sets of HS's are installed, each containing 8 miniature HS's (Fig. 1, only sensors on one leg are shown). The details of the samples' description and preparation can be found in [2].

The SULTAN background field is inhomogeneous along the length of the conductor. Its peak is located at the part of the conductor between the potentials V13 and V9. All experiments were carried out at a nominal peak field of $10 \mathrm{~T}$.

All HS arrays are installed on the same straight leg of the sample. The first set (HS12-18) is placed close to the joints. An angle of $30^{\circ}$ between the normal to the conductor surface and the HS plane is selected to minimize the influence of the experimental errors on the results of the currents reconstruction (Fig. 1). Purely considered as a mathematical problem, the conditioning number of the matrix, whose elements are partly functions of the HS orientation, can be minimized by varying this angle [4], [5]. The second set is located on the section of the conductor in the high field region (HS21-28).

In this set, the HS planes are oriented parallel to the background magnetic-field vector in order to minimize its influence on the self field measurements (Fig. 1). The third (HS31-38) and fourth (HS41-48) arrays are installed on the conductor section in the intermediate field zone and on the U-bend correspondingly. The orientation of the HS's in these sets is identical to those of the set in peak field. 



Fig. 2. Magnification of the HS traces near the joints and in high field zone from a $V-T$ test on conductor $A$ before cycling. A constant current of $10 \mathrm{kA}$ is set in the conductor. The current ramp rate is $213 \mathrm{~A} / \mathrm{s}$. Also shown are the temperature and voltage over the part of conductor in high field.

All the HS's were procured with the calibration sheets. After the HS's had been assembled in the array holders, they were recalibrated again. The same sets of HS's were used for both samples $A$ and $B$ for identical locations. The analysis of the self field measurements reported here is performed on the HS's in the high field region and near the joints, as they are located in both lowest and highest background field. The sample was charged with transport current by a transformer method (in which the sample is the part of the secondary winding).

\section{EXPERIMENTAL RESULTS AND THEIR INTERPRETATION}

\section{A. Self Field Measurements Close to Joints}

The goal of the $V-T$ test is to determine the current sharing temperature at different levels of transport current. This type of test is performed twice-before cycling load and after 3400 cycles.

A magnification of the HS traces near the joints region of conductor $A$ during the cycle \#15 is shown in Fig. 2. During charging the HS's signals were linear with the transport current. As soon as transport current becomes constant in the conductor (10 kA after ramping up with $213 \mathrm{~A} / \mathrm{s})$, the signals from the HS's remain unchanged $(<1 \%)$ during $60 \mathrm{~s}$. Later on, when the temperature is raised by a heater, a clear change in the signals up to $2.5 \%$ for some of the HS is observed until voltage takeoff (during $\sim 100 \mathrm{~s}$ ). The redistribution of the currents is initiated by the growth of the resistance in the conductor section at high field. These results are in good quantitative agreement with the results from the experiments on the SeCRETS long sample [3].

After 3400 load cycles, the $V-T$ test is repeated but this time with a higher ramp rate of $520 \mathrm{~A} / \mathrm{s}$ and with the long "plateau time"- the time interval between the instants when the constant current is set and when the temperature starts raising. The evolution of the HS signals is shown in Fig. 3. The characteristic time of the transient process determined by exponential fitting of the experimental curves, before reaching a constant level, are in the range of $250-350 \mathrm{~s}$.

Fig. 3. Magnification of the HS traces near the joints and in high field zone from a $V-T$ test on conductor $A$ after cycling. A constant current of $8 \mathrm{kA}$ is set in the conductor. The current ramp rate is $512 \mathrm{~A} / \mathrm{s}$. Also shown are the temperature and voltage over the part of conductor in high field.

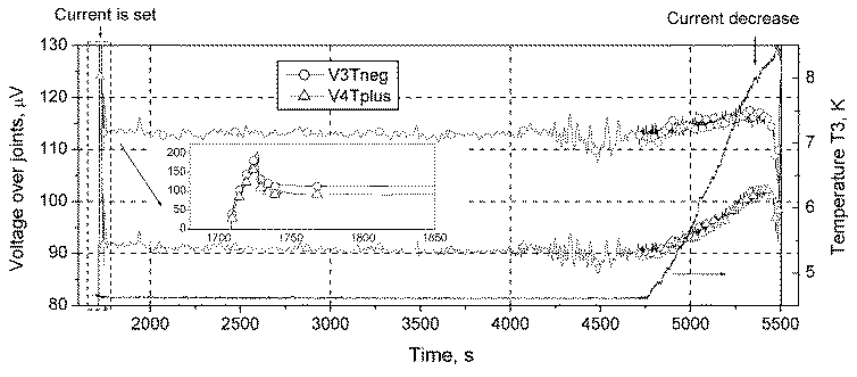

Fig. 4. Voltages in both joints of conductor $A$ during $V-T$ test after 3400 cycles at $8 \mathrm{kA}$. The inset picture demonstrates the voltage behavior just before the current ramping ends.

Just after fast current ramping, the current distribution is driven mainly inductively and is expected to be nearly uniform. During the "plateau time," the currents in the conductor redistribute according to the resistances between the individual strands in the cable (quite high, $\sim 210 \mathrm{n} \Omega \mathrm{m}$ ) and in the joint regions. The final distribution must satisfy the condition of a minimum voltage across the joints. If the redistribution driven by the joints resistance is not uniform, then the currents will be forced to redistribute again when the temperature starts rising in the conductor and the current sharing in the high field zone occurs. As a result, the voltage on the joints will increase again.

In Fig. 4 the voltages on both joints are shown for the same cycle as in Fig. 3. After the current is set constant, the voltages on the joints decay with a short characteristic time constant of about $10 \mathrm{~s}$. The voltage over the joints increases when current sharing occurs in the high field section of the conductor due to the temperature raise.

The suggested scenario seems in agreement with the experimental data. However, in spite of the fast initial current ramp, during the long current plateau time the voltages on the joints barely changed (Fig. 4). This is somehow in contradiction with the evolution of the signals from the HS's, which changed even more than during the temperature rise in the same run. Therefore 


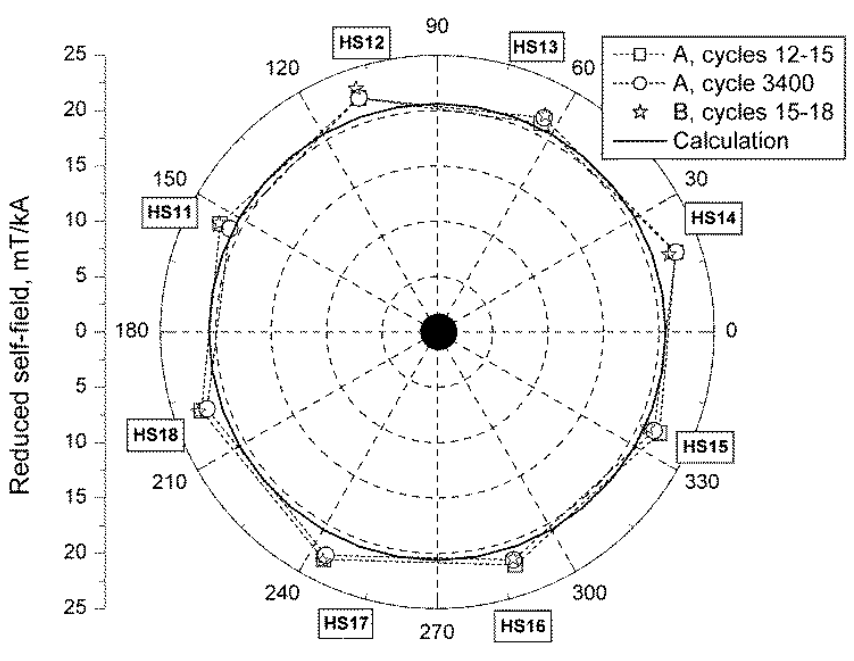

Fig. 5. Reduced self field near the joints of conductor $A$ (before and after cycling) and conductor $B$ (before cycling) at voltage takeoff in $V-T$ test. The lines connecting the data are only for visual aid. The calculated values, assuming a uniform current distribution are also shown.

a larger change in the joints voltage would be expected during the constant current plateau, than during the temperature rise.

In terms of the HS traces, the suggested scenario means that during the plateau time the signals are expected to deviate from their initial value just after ramping. Then, during the temperature increase, the HS traces are expected to return into the direction of their initial value.

The final shape of the HS traces can be characterized as "U-shape." For example, in experiments with the ITER CSIC (Central Solenoid Insert Conductor) the HS traces of the "U-shape" were clearly observed [6].

However, in Fig. 3 it can be seen that some of the traces (for example HS13 and HS15) have a different shape, which could be characterized as an "S-shape." This suggests that already immediately after the ramp, the currents are not distributed uniformly in the conductor or that the final current distribution is not uniform. This assumption will be discussed in Section III-B.

The maximum change of the self field signals during the long current plateau time is for the HS11 and amounts to $\sim 8 \%$. The signals from the other HS's changed within 1-5\%. According to the model proposed in [6], this means a maximum change of the final stage sub-bundle current of $\sim 25 \%$ or $500 \mathrm{~A}$ at a total current of $8 \mathrm{kA}$ in the conductor. The difference between the values at the instant when temperature starts rising and just before voltage takeoff occurs, amounts up to $2.5 \%$ and gives a change of $\sim 160 \mathrm{~A}$ in the current of final stage sub-bundle.

The averaged reduced self field data at voltage takeoff (self field divided by total current) from both experimental series before and after cycling are plotted in Fig. 5. On this polar plot, the magnitude of the radius represents the self field value and the angle corresponds to the annular position of a HS. Also shown in Fig. 5 are the calculated values of the self field assuming a uniform current distribution in the conductor. The calculated self field pattern is in good qualitative agreement with the experimental data but still deviates in the absolute sense.

The calculated data are $2.5 \%$ to $13 \%$ lower than the ones obtained experimentally for the different HS's.
The misalignments of the HS's are considered to be a major reason for this difference [7]. It is not likely that this difference is caused by a nonuniform current distribution in the conductor because in this case a variation of the experimental data around calculated ones would be expected.

However, there is a considerable difference between the data from $V-T$ tests before and after cycling. A maximum difference of $4.6 \%$ between the values before and after cycling is found for HS11. It means the maximum change of $\sim 15 \%$ in the current of final stage sub-bundle. It should be remarked that the conditions of the $V-T$ tests before and after cycling are different $(d I / d t$, current level and time scale), which can also introduce some error in the HS signals interpretation.

\section{B. Self Field Measurements in the High Field Region}

Unlike the HS close to the joints, the HS's in high field region demonstrated strongly nonlinear behavior with transport current during charging. In the $V-T$ experiments on conductor $A$ performed before cycling at $10 \mathrm{kA}$ (Fig. 2), the HS signals in the high field region, changed less than $1 \%$ after the current has been set constant. A change up to 5\% for some of the HS's was observed during the temperature increase in the conductor. This change is larger than for the HS signals near the joints.

After 3400 cycles, during the $V-T$ run with long plateau time, the maximum change of $30 \%$ was observed for HS26 during long plateau time (Fig. 3). The signals from the other HS's changed within $8-12 \%$. This difference is much larger again than observed in the HS's near the joints (Fig. 2). According to the model this means a change of the current in final stage sub-bundle by factor of 2.5. It suggests that along with the current redistribution due to nonuniform joints, some local redistribution may also take place.

During the temperature rise, the signals only changed up to $5 \%$. The characteristic time constants of the transient process for the HS signals in the high field are of the same order of magnitude as for the signals from the HS near the joints, i.e., $150-350 \mathrm{~s}$.

In Fig. 6 the reduced self field data from the $V-T$ tests before and after cycling, as measured by the HS's in high field zone just before voltage takeoff, are shown. The difference between the experimental data and calculated ones in this case is significant. The largest difference is observed for HS26 and HS24 (more than twice). According to the applied model [6], this difference cannot be explained without assuming opposite current directions in some of the strand bundles (in relation to the total transport current). However, this solution is considered to be not viable because just before voltage takeoff the current is expected to be virtually balanced. The reason for such a discrepancy is not clear and so errors from experimental kind or in terms of modeling cannot be excluded at this point.

In this context it should be mentioned, that the discrepancy between the experimental and calculated values in the present experiment is larger than observed in the experiments with the long SeCRETS samples ( $25 \%$ for conductor $A$ ). It was suggested in [3] that this deviation could be caused by the self field effect, causing a nonuniform current distribution in the conductor. From the results of the present $V-T$ experiments this hypothesis may be disproved by the fact that a significant difference between calculated and experimental data is already ob- 




Fig. 6. Reduced self field in high field region of conductor $A$ (before and after cycling) and conductor $B$ (before cycling) at voltage takeoff in $V-T$ test. The calculated values, assuming a uniform current distribution are also shown. The error bars show the deviation from average in different runs.

served at currents far below current sharing in the high field region. Moreover, the change of the HS signals during the temperature rise does not exceed $5 \%$.

The difference between the self field patterns before and after the cycling is much bigger compared to that near the joints (Fig. 6). The most likely explanation is that the self field profile has changed with cycling. This change can possibly be caused by the local damage of the strands due to Lorenz force [2]. Nevertheless, it cannot be fully excluded that the different experimental conditions of the runs play some role as well, and so it comes up again that is important to compare runs with identical experimental conditions. Unfortunately these runs were not performed.

It should be remarked that the self field profiles, measured by the individual HS's in all four locations just after the ramping of the current, have all become different with cycling. However, the largest difference between the data (immediately after ramping) is observed for HS's in the high field zone, i.e., in the location of maximum load. The timescale of the profiles are all in a good agreement.

\section{Results From Conductor B}

A few $V-T$ tests performed on conductor $B$ before cycling are available for analysis. In Fig. 5, the averaged reduced self field data just before voltage takeoff for the HS's near the joints are shown for both conductors. The self field pattern near the joints for both conductors is similar and in agreement with the calculated data. The absolute difference between the data for particular HS between both conductors can be up to $4 \%$. The difference between the experimental data for conductor $B$ and the calculated values remains several percents and comparable to what is observed for conductor $A$.

In Fig. 6 a similar comparison is done for the experimental self field data between conductors $A$ and $B$ in the high field region. In this case a qualitative as well as a quantitative difference between two sets of data is observed. The largest deviation of $32 \%$ for the data from HS26 and $20 \%$ for HS24 is observed for conductor $A$.
For conductor $B$ there is still a significant difference for HS21 and HS28 with respect to the calculated data. If the large deviation between the experimental data and the calculated ones observed for conductor $A$ can be explained by an experimental error due to unknown behavior of the HS in high parallel field, then, the same behavior would be expected for the same sets of HS's in the experiments with conductor $B$.

This suggests that the measured self field pattern in the high field region would be a result of the local current distribution in the conductor. Then, according to the applied model [6], this distribution is extremely nonuniform. Another suggestion of an experimental error, which may not be totally excluded on forehand, is that the orientation of the HS in the background field did not remain exactly identical for both conductor tests.

\section{SUMMARY}

Both sets of HS arrays at different locations along the sample demonstrate similar behavior in time with identical characteristic time constants, but different changes in self field magnitudes.

After a fast ramp of the current, transient phenomena can be observed with characteristic time constants in a range of roughly 150-350 s for various HS's.

Clear changes in the HS signals are observed when the temperature is increased in the conductor toward the current sharing regime in the high field region. A current redistribution is enforced due to an existing nonuniform current distribution.

Some quite extreme results have been observed with respect to possible interpretations of the current distribution reconstructed from the self field traces in the high field zone, especially for conductor $A$. Although the possible errors from experimental kind and from interpretative kind cannot be fully excluded, this can be considered as an indication of strong nonuniform current redistribution.

The influence of cyclic load on the self field profile near the joints seems insignificant. Conversely, there is a change up to $20 \%$ in some of HS traces in the high field zone and so there is a strong suggestion that the properties of the conductor have changed locally during cycling.

\section{REFERENCES}

[1] P. Bruzzone, A. M. Fuchs, B. Stepanov, G. Vecsey, and E. Zapretelina, "Test results of SeCRETS, a stability experiment about segregated copper in CICC," IEEE Trans. on Appl. Superc., vol. 11, pp. 2018-2021, 2001.

[2] P. Bruzzone, R. Wesche, and B. Stepanov, "The voltage/current characteristic ( $n$ value) of the cable-in-conduit conductors for fusion," in this conference.

[3] Yu. Ilyin, A. Nijhuis, H. H. J. ten Kate, and P. Bruzzone, "Self field measurements by Hall sensors on the SeCRETS long sample CICC's in SULTAN," IEEE Trans. on Appl. Superc., vol. 12, no. 1, pp. 1667-71.

[4] F. Bellina, P. Bettini, and F. Trevisan, "Analysis of the optimal location of magnetic field probes for determination of a current distribution inside the s/c cables," IEEE Trans. on Appl. Superc., vol. 11, pp. 2379-82.

[5] P. Bruzzone, A. Formisano, and R. Martone, "Optimal magnetic probes location for current reconstruction in multistrand superconducting cables," IEEE Trans. on Mag., vol. 38, p. 1057, 2002.

[6] A. Nijhuis, Yu. Ilyin, and H. H. J. ten Kate, "Analysis of the current distribution in the ITER CS-insert model coil conductor by self field measurements,", vol. 12, no. 1, pp. 1675-79, 2002.

[7] A. Nijhuis and Yu. Ilyin, "Current distribution: Reconstruction from self-field profile and impact on cable $n$-value,", report EFDA-00/552, 2001. 